\title{
Regional Neuronal Network Failure and Cognition in Late-Onset Sporadic Alzheimer Disease
}

\author{
S.F. Carter, K.V. Embleton, J.M. Anton-Rodriguez, A. Burns, M.A.L. Ralph, and K. Herholz
}

\begin{abstract}
BACKGROUND AND PURPOSE: The severe cognitive deficits in Alzheimer disease are associated with structural lesions in gray and white matter in addition to changes in synaptic function. The current investigation studied the breakdown of the structure and function in regional networks involving the Papez circuit and extended neocortical association areas.
\end{abstract}

MATERIALS AND METHODS: Cortical volumetric and diffusion tensor imaging (3T MR imaging), positron-emission tomography with ${ }^{18} \mathrm{~F}$ fluorodeoxyglucose on a high-resolution research tomograph, and comprehensive neuropsychological assessments were performed in patients with late-onset sporadic Alzheimer disease, those with mild cognitive impairment, and elderly healthy controls.

RESULTS: Atrophy of the medial temporal lobes was the strongest and most consistent abnormality in patients with mild cognitive impairment and Alzheimer disease. Atrophy in the temporal, frontal, and parietal regions was most strongly related to episodic memory deficits, while deficits in semantic cognition were also strongly related to reductions of glucose metabolism in the posterior cingulate cortex and temporoparietal regions. Changes in fractional anisotropy within white matter tracts, particularly in the left cingulum bundle, uncinate fasciculus, superior longitudinal fasciculus, and inferior fronto-occipital fasciculus, were significantly associated with the cognitive deficits in multiple regression analyses. Posterior cingulate and orbitofrontal metabolic deficits appeared to be related to microstructural changes in projecting white matter tracts.

CONCLUSIONS: Many lesioned network components within the Papez circuit and extended neocortical association areas were significantly associated with cognitive dysfunction in both mild cognitive impairment and late-onset sporadic Alzheimer disease. Hippocampal atrophy was the most prominent lesion, with associated impairment of the uncinate and cingulum white matter microstructures and hippocampal and posterior cingulate metabolic impairment.

ABBREVIATIONS: ACE-R = Addenbrooke's Cognitive Examination-Revised; $\mathrm{AD}=$ Alzheimer disease; $\mathrm{FA}=$ fractional anisotropy; $\mathrm{HCMP}=$ hippocampus; $\mathrm{MCI}=$ mild cognitive impairment; MTL = medial temporal lobe; $\mathrm{PCC}=$ posterior cingulate cortex; TBSS $=$ Tract-Based Spatial Statistics

$\mathbf{N}$ ormal cognition is dependent on the activation of and communication among distributed neuronal networks. Papez ${ }^{1}$ highlighted the importance of the medial temporal lobe (MTL) circuit, which takes his name, in cognitive function. Further work

Received April 9, 2013; accepted after revision July 2.

From the Wolfson Molecular Imaging Centre (S.F.C., J.M.A.-R., K.H.), Institute of Population Health, Neuroscience and Aphasia Research Unit (S.F.C., M.A.L.R.), School of Psychological Sciences (K.V.E.), and Institute of Brain, Behaviour and Mental Health (A.B., K.H.), University of Manchester, Manchester, UK; and Alzheimer Neurobiology Centre (S.F.C.), Department of Neurobiology, Care Sciences and Society, Karolinska Institutet, Stockholm, Sweden.

S.F.C. was supported by a Medical Research Council capacity building studentship (to M.A.L.R., K.H., and A.B.).

Please address correspondence to Prof. Karl Herholz, MD, Wolfson Molecular Imaging Centre, University of Manchester, 27 Palatine Rd, Withington, Manchester, M20 3LJ, UK; e-mail: karl.herholz@manchester.ac.uk

- Indicates open access to non-subscribers at www.ajnr.org

http://dx.doi.org/10.3174/ajnr.A3895 by Maclean ${ }^{2}$ and others ${ }^{3,4}$ extended the Papez circuit. This extended MTL network includes the hippocampus (HCMP), anterior thalamic nuclei, mammillary bodies, fornix, fimbria, entorhinal cortex, parahippocampal cortex, posterior cingulate cortex (PCC), and anterior temporal lobe. White matter fasciculi, including the cingulum bundle, uncinate fasciculus, and superior and inferior longitudinal fasciculi, connect neocortical association areas in frontal, temporal, and parietal regions. This whole network is integral for episodic memory function, ${ }^{4}$ semantic cognition, ${ }^{5}$ executive function, ${ }^{6}$ and behavioral performance. ${ }^{7}$

The MTL-memory network is severely affected in Alzheimer disease (AD) by the formation of fibrillar amyloid- $\beta$ deposits (mostly in neocortical association areas) and neurofibrillary tangles (mostly in the MTL). ${ }^{8-11}$ Neuroimaging techniques have studied the macroscopic structural and functional changes associated with $\mathrm{AD}$ pathology and how they relate to cognitive im- 

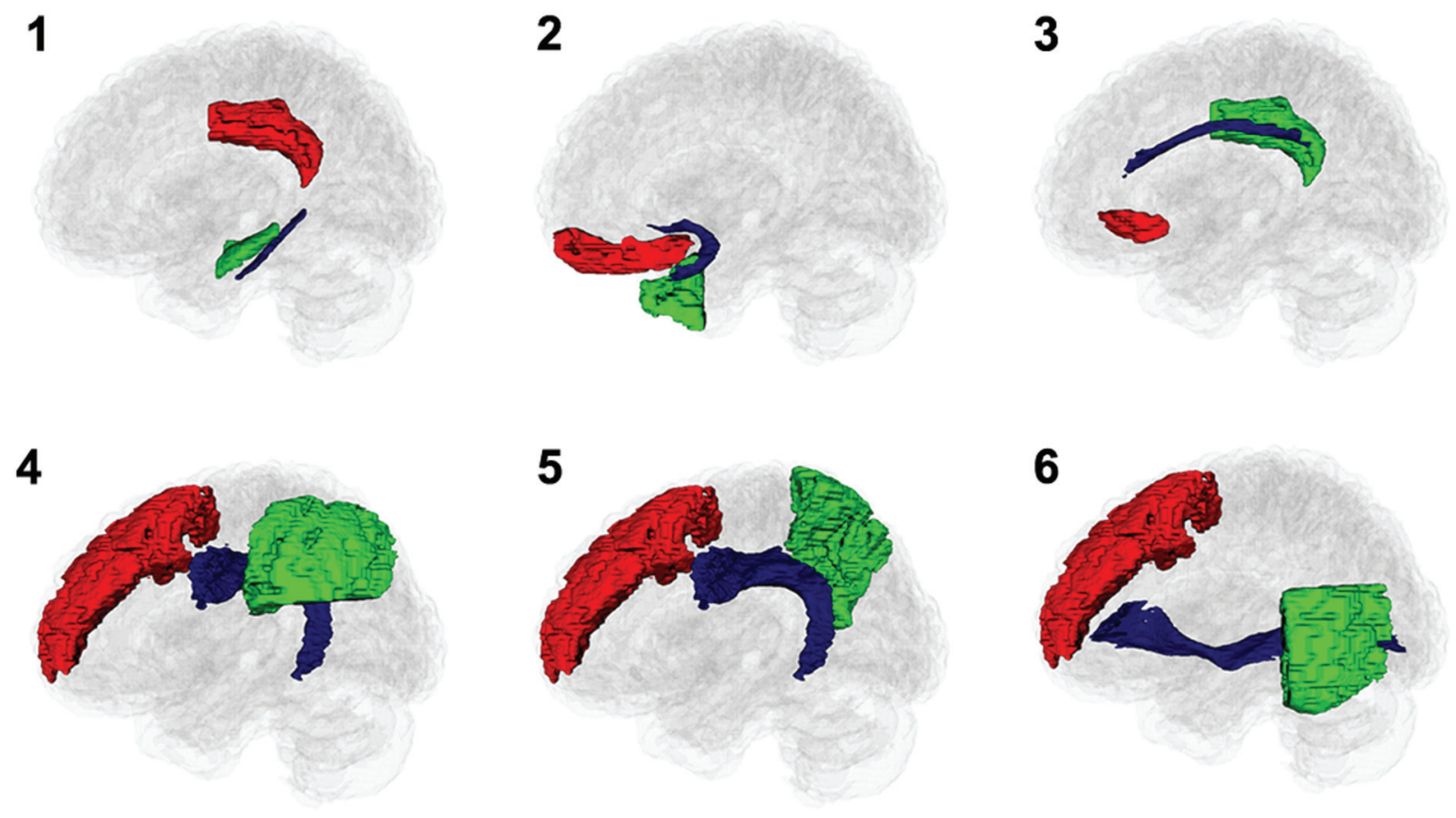

FIG 1. The neocortical networks investigated (see Table 2 for components).

pairment in vivo. Morphometric techniques by using MR imaging, positron-emission tomography with ${ }^{18} \mathrm{~F}$ fluorodeoxyglucose, and diffusion tensor imaging have demonstrated gray matter atrophy of the MTL, which is closely related to memory impairment and disease progression, ${ }^{12}$ hypometabolism of the PCC and lateral temporoparietal areas, and degeneration of WM fasciculi (particularly the cingulum bundle), respectively (see Chua et al, ${ }^{13}$ Frisoni et al, ${ }^{14}$ and Herholz ${ }^{15}$ for reviews). The combination of neuroimaging methods clearly demonstrates neuronal network breakdown in AD (see Filippi and Agosta ${ }^{16}$ for a review). Furthermore, neuroimaging studies ${ }^{17-19}$ have indicated that there is a significant sequential process that leads to neocortical disconnection among primary lesions in the HCMP, impaired WM pathways (eg, cingulum bundle), and tissue function at connected neocortical nodes (eg, hypometabolism at the PCC). Additionally neuropsychological investigations have suggested that episodic and semantic cognition impairments are typical and prominent in prodromal and manifest $\mathrm{AD} .^{20,21}$

The main objective of the current investigation, therefore, was to examine the extended MTL-memory network by using multitechnique neuroimaging in a cross-sectional design incorporating healthy controls and patients with mild cognitive impairment (MCI) and mild AD. In particular, measurements of regional neurodegeneration (MR imaging morphometry), regional hypometabolism (FDG-PET), and integrity of WM fasciculi (diffusion tensor imaging) were performed in 6 neocortical networks (Fig 1). The disease-related changes within each network component were related, via multiple regression analysis, to neuropsychological performance. The primary networks of interest were network 1 (HCMP-cingulum-PCC) and network 2 (anterior tem- poral lobe-uncinate-medial orbitofrontal cortex), which have been associated with episodic and semantic cognition, respectively. ${ }^{4,5,22,23}$ If neuronal loss in mesial and anterior temporal lobe structures and impaired integrity of associated fibers lead to neocortical disconnection and hypometabolism of projection sites, we hypothesized that fractional anisotropy (FA) in the respective WM fasciculi would be significantly correlated to GM hypometabolism and would significantly contribute to cognitive dysfunction in regression analyses.

To achieve our objectives, we used a dedicated high-resolution PET brain scanner ${ }^{24}$; PET data were coregistered with 3T MR imaging data, providing excellent precision and contrast for morphometry and DTI. Combining these modalities allowed us to dissect the MTL-memory network with previously unavailable resolution.

\section{MATERIALS AND METHODS Participants}

Thirty-two participants (13 healthy controls and 20 patients, 12 with MCI and 8 with mild AD) participated in this investigation, which was approved by the Central Manchester Research Ethics Committee (UK). All patients (and their caregivers/next of kin) gave signed, informed consent. All patients were recruited from an old-age memory clinic at Wythenshawe Hospital, Manchester, UK. Patients with mild AD were classified as having a Mini- Mental State Examination score typically between 20 and 25 and fulfilled the criteria developed by the National Institute of Neurologic and Communicative Disorders and Stroke-Alzheimer Disease and Related Disorders Association. ${ }^{25}$ Patients with MCI fulfilled the criteria established by Petersen ${ }^{26}$ and were best char- 
Table 1: Basic descriptive statistics and neuropsychological scores ${ }^{\mathrm{a}}$

\begin{tabular}{|c|c|c|c|c|c|c|}
\hline & \multicolumn{2}{|c|}{$\mathrm{HC}$} & \multicolumn{2}{|c|}{$\mathrm{MCl}$} & \multicolumn{2}{|c|}{$A D$} \\
\hline & Mean & SD & Mean & SD & Mean & SD \\
\hline Age (yr) & $69.6^{\mathrm{b}}$ & 5.5 & 74.1 & 6.4 & $77.8^{c}$ & 5.0 \\
\hline $\operatorname{Sex}(M / F)$ & $3: 8$ & & $9: 2$ & & $5: 3$ & \\
\hline Years of education & 13 & 3.2 & 13.5 & 4.1 & 11.5 & 3.2 \\
\hline MMSE score & $29.6^{b}$ & 0.5 & 28.6 & 1.3 & $24.9^{c}$ & 2.8 \\
\hline$A P O E \in 4$ & $1 / 10$ & & $5 / 8$ & & $7 / 8$ & \\
\hline $\operatorname{TIV}\left(\mathrm{cm}^{3}\right)$ & 1700 & 160 & 1800 & 120 & 1800 & 210 \\
\hline Total ACE-R & $94.6^{\mathrm{b}, \mathrm{d}}$ & 4.3 & $88.7^{\mathrm{b}, \mathrm{c}}$ & 6.1 & $73.3^{\mathrm{c}, \mathrm{d}}$ & 7.9 \\
\hline ACE-R Memory & $23.7^{b, d}$ & 2.2 & $19.5^{\mathrm{b}, \mathrm{c}}$ & 3.6 & $11.1^{\mathrm{c}, \mathrm{d}}$ & 2.9 \\
\hline Category Fluency & $100^{b, d}$ & 12.6 & $77.8^{\mathrm{b}, \mathrm{c}}$ & 17.5 & $55.1^{c, d}$ & 15.4 \\
\hline ACE-R Attention & $18^{\mathrm{b}}$ & 0.0 & 17.8 & 0.4 & $16^{c}$ & 1.9 \\
\hline
\end{tabular}

Note:- $-\mathrm{HC}$ indicates healthy controls; APOE $\epsilon 4$ indicates the number of participants carrying at least $1 \epsilon 4$ allele (APOE data were unavailable from 1 healthy control and 3 patients with MCI); MMSE, Mini-Mental State Examination; TIV, total intracranial volume.

${ }^{a}$ Superscript symbols indicate significant pair-wise comparisons revealed by post hoc testing (Tamhane T2, $\left.P=.05\right)$. There was a significant group difference for age, $F(2,27)=$ 4.5, $P<.05$; MMSE score, $F(2,27)=20.2, P<.001$; total ACE-R, $F(2,27)=29.9, P<.001$; ACE-R Memory, $F(2,27)=42.2, P<.001$; Category Fluency, F(2, 27) = 20.1, $P<.001$; ACE-R Attention, $F(2,27)=11.4, P<.001$.

bignificantly different from AD.

c Significantly different from HC.

d Significantly different from $\mathrm{MCl}$.

acterized as amnestic multiple domain; they had Mini-Mental State Examination scores of $\geq 26$. All participants underwent an extensive neuropsychological investigation (a detailed description of the neuropsychological test battery can be found in Carter et $\mathrm{al}^{20}$ ), and all investigations, including neuroimaging, were completed within a 6-week time frame. A summary of the basic participant information is found in Table 1, and more details are provided in the Appendix. There was a small but significant difference in age among groups, and we, therefore, used age as a covariate in all statistical analyses.

\section{MR Imaging and PET Acquisition}

All MR imaging was performed on the same 3T Achieva scanner (Philips Healthcare, Best, Netherlands) by using an 8-element sensitivity encoding head coil. A T1-weighted inversion recovery acquisition was acquired parallel to the anterior/posterior commissure line with a 3D acquisition. Diffusion-weighted imaging was performed by using a pulsed gradient spin-echo EPI sequence.

All PET scans were obtained on an ECAT high-resolution research tomograph (HRRT; CTI-Seimens, Knoxville, Tennessee). ${ }^{28,29}$ PET images were reconstructed from data acquired 20- to 60-minutes postinjection of $340 \mathrm{MBq}$ of ${ }^{18} \mathrm{~F}$ fluorodeoxyglucose.

Details of the data acquisition are available in the Appendix.

\section{Image Processing and Analysis Methods}

A detailed description of the image-processing and analysis methods can be found in the Appendix. All T1-weighted images were segmented by using SPM5 (Wellcome Department of Imaging Neuroscience, London, UK) into gray matter and white matter tissue classes. These probabilistic tissue classes were then entered into the DARTEL (http://www.fil.ion.ucl.ac.uk/spm/) toolbox to form a population template. All probabilistic GM images and corresponding coregistered FDG images were then spatially normalized by using the DARTEL warp fields. The FDG images were intensity-normalized to the median uptake in each individual's precentral gyrus. All DARTEL spatially normalized and modulated GM and spatially normalized and intensity-normalized FDG images were an- alyzed by using the general linear model in SPM5 with an 8-mm filter kernel. Regression analyses were performed on the imaging data with the neuropsychological assessments that had revealed the largest group effect (Addenbrooke's Cognitive ExaminationRevised [ACE-R] memory, Category fluency-total score and ACE-R Attention and Orientation; Table 2) to determine the regional relationship among cognition, GM volume, and FDG uptake.

In addition to the SPM5 analysis, tract-based spatial statistics (TBSS) (Version 1.2, http://fsl.fmrib.ox.ac.uk/fsl/fslwiki/TBSS) were performed on the DTI data. Diffusion-weighted data were corrected for inhomogeneity artifacts following the procedure outlined in Embleton et al. ${ }^{34}$ Fractional anisotropy images were compared among the 3 different groups. For each contrast, a null distribution was constructed from 5000 random permutations. Significance was tested at $P<.05$. The threshold-free cluster enhancement method ${ }^{36}$ was used during analysis, and results were corrected for multiple comparisons.

To supplement the SPM5 and TBSS voxelwise analyses, we also performed region-of-interest analyses for each technique (structural MR imaging, DTI, and FDG-PET).

\section{RESULTS}

\section{Neuropsychological Results}

Multivariate analysis with all neuropsychological tests entered as dependent variables and age as a covariate was used to compare the cognitive status among groups. There was a significant effect of group $(P=<.05)$, but there was no overall effect of age in the model. Many neuropsychological tests revealed highly significant group effects in univariate analysis (Table 2).

The overall score obtained by the Addenbrooke's Cognitive Examination-Revised showed highly significant differences among groups $(P=<.001)$, with subtests indicating that episodic memory was the most prominently impaired cognitive domain, followed by semantic cognition. Attention/executive functions were slightly impaired, with relative sparing of visuospatial functions. 
Table 2: Means and SDs for neuropsychological test scores ${ }^{a}$

\begin{tabular}{|c|c|c|c|c|c|c|c|c|}
\hline \multirow[b]{2}{*}{ Test } & \multicolumn{2}{|c|}{$\mathrm{HC}$} & \multicolumn{2}{|c|}{$\mathrm{MCl}$} & \multicolumn{2}{|c|}{$A D$} & \multirow[b]{2}{*}{$F(2,27)$} & \multirow[b]{2}{*}{$P$ Value } \\
\hline & Mean & SD & Mean & SD & Mean & SD & & \\
\hline Total ACE-R & $94.6^{\mathrm{b}, \mathrm{c}}$ & 4.3 & $88.7^{c, d}$ & 6.1 & $73.3^{\mathrm{b}, \mathrm{d}}$ & 7.9 & 29.9 & $<.001$ \\
\hline \multicolumn{9}{|l|}{ Episodic Memory } \\
\hline ACE-R-Memory & $23.7^{b, c}$ & 2.2 & $19.5^{\mathrm{c}, \mathrm{d}}$ & 3.6 & $11.1^{\mathrm{b}, \mathrm{d}}$ & 2.9 & 42.2 & $<.001$ \\
\hline FPT & $91.8^{c}$ & 4.9 & $82.7^{c}$ & 10.6 & $65.6^{\mathrm{b}, \mathrm{d}}$ & 7.9 & 24.0 & $<.001$ \\
\hline FPT-Familiarity & 19.2 & 1.1 & 18.6 & 1.4 & 18 & 2.0 & 1.5 & NS \\
\hline FPT-Recognition & 37.2 & 5.7 & 38.2 & 2.3 & 34.1 & 5.0 & 1.9 & NS \\
\hline FPT-Placing & $18.9^{c}$ & 1.6 & $15.0^{c}$ & 4.4 & $8.6^{b, d}$ & 5.4 & 13.6 & $<.001$ \\
\hline STOREC-Immediate & $47.5^{c}$ & 7.9 & $37.5^{c}$ & 12.7 & $16.9^{b, d}$ & 8.8 & 21.4 & $<.001$ \\
\hline STOREC-Delayed & $29.9^{c}$ & 6.8 & $22.2^{c}$ & 13.4 & $2.4^{\mathrm{b}, \mathrm{d}}$ & 3.5 & 20.9 & $<.001$ \\
\hline RCFT-Delayed & $18.9^{c}$ & 7.3 & $15.6^{\mathrm{c}}$ & 5.1 & $6.5^{\mathrm{b}, \mathrm{d}}$ & 5.6 & 9.7 & .001 \\
\hline CVLT & $10.5^{c}$ & 2.8 & 7.7 & 2.4 & $5.1^{d}$ & 2.2 & 10.9 & $<.001$ \\
\hline CVLT-Delayed & $11.6^{\mathrm{b}, \mathrm{c}}$ & 3.3 & $6.7^{\mathrm{c}, \mathrm{d}}$ & 4.5 & $1.0^{\mathrm{b}, \mathrm{d}}$ & 1.2 & 21.6 & $<.001$ \\
\hline Digit Span-Forwards & 5.9 & 1.0 & 5.7 & 1.4 & 5.9 & 1.4 & .06 & NS \\
\hline \multicolumn{9}{|l|}{ Language/Semantic Memory } \\
\hline ACE-R-Fluency & 12.4 & 1.9 & 10.6 & 2.1 & 10.3 & 3.2 & 2.3 & NS \\
\hline ACE-R-Language & 24.9 & 1.5 & 25.0 & 1.5 & 23.4 & 3.4 & 1.6 & NS \\
\hline FPT-Naming & $15.6^{\mathrm{c}}$ & 3.7 & $10.9^{c}$ & 6.0 & $4.8^{\mathrm{b}, \mathrm{d}}$ & 3.7 & 11.9 & $<.001$ \\
\hline FAS Fluency-Total & $52.8^{c}$ & 12.5 & 44.6 & 10.7 & $33.5^{\mathrm{d}}$ & 13.1 & 6.0 & $<.01$ \\
\hline Category Fluency-Total & $100^{\mathrm{b}, \mathrm{c}}$ & 12.6 & $77.8^{\mathrm{c}, \mathrm{d}}$ & 17.5 & $55.1^{\mathrm{b}, \mathrm{d}}$ & 15.4 & 20.1 & $<.001$ \\
\hline Graded Naming & $26.2^{c}$ & 1.8 & 22.8 & 4.1 & $16.6^{\mathrm{d}}$ & 6.1 & 12.6 & $<.001$ \\
\hline \multicolumn{9}{|l|}{ Attention/Executive } \\
\hline ACE-R-Attention and Orientation & $18^{c}$ & 0.0 & 17.8 & 0.4 & $16^{d}$ & 1.9 & 11.4 & $<.001$ \\
\hline Digit Span-Backwards & 3.6 & 1.4 & 4.2 & 1.4 & 3.9 & 1.4 & 6 & NS \\
\hline TEA-Map Search & $58.2^{c}$ & 9.3 & 45.0 & 16.1 & $32.9^{d}$ & 12.7 & 8.9 & .001 \\
\hline TEA-Elevator counting distraction & $8.2^{c}$ & 2.4 & 8.3 & 2.0 & $7.6^{\mathrm{d}}$ & 2.8 & .2 & NS \\
\hline STROOP-Words & $94.1^{b, c}$ & 13.7 & $75.9^{d}$ & 17.1 & $65^{d}$ & 19.9 & 7.4 & $<.01$ \\
\hline STROOP-Colors & $36.9^{c}$ & 10.4 & 26.3 & 10.4 & $17.9^{d}$ & 11.1 & 7.7 & $<.01$ \\
\hline STROOP-Percentage & 39.3 & 10.9 & 34.8 & 12.7 & 32.2 & 25.4 & .5 & NS \\
\hline WCST-Category Shifts & 2.7 & 1.7 & 2.5 & 1.4 & 1.6 & 1.4 & 1.3 & NS \\
\hline \multicolumn{9}{|l|}{ Visuospatial } \\
\hline RCFT-Copy & 34.1 & 2.6 & 33.0 & 5.3 & 30.4 & 8.1 & 1.1 & NS \\
\hline ACE-R-Visuospatial & 15.5 & 0.9 & 15.7 & 0.6 & 15.3 & 1.8 & .4 & NS \\
\hline
\end{tabular}

Note:-HC indicates healthy controls; NS, not significant; FPT, Face Place Test; STOREC, story recall; RCFT, Rey Complex Figure Test; CVLT, California Verbal Learning Test; TEA Test of Everyday Attention; WSCT, Wisconsin Card Sorting Test.

a Superscript letters indicate significant pair-wise comparisons revealed by post hoc testing (Tamhane T2, $P=.05$ ).

b Significantly different from $\mathrm{MCl}$.

'Significantly different from AD.

d Significantly different from HC.

\section{SPM5 Results}

Separate regression analyses were performed with both the MR imaging and PET data by using the whole participant population with the ACE-R Memory, Category Fluency total, and ACE-R Attention and Orientation neuropsychological test scores. Significant regional morphologic correlations across the population were revealed for both the ACE Memory (episodic memory) and total Category Fluency score but not for the ACE-R Attention and Orientation. Clusters that had significant GM morphologic correlations with the cognitive tasks were located predominantly in the MTL (HCMP and parahippocampal cortex), anterolateral temporal, PCC, and medial frontal regions.

The same methodology applied to FDG data revealed significant regional correlations across the population for the ACE-R Memory and total Category Fluency score but not for the ACE-R Attention and Orientation. Clusters that had significant FDGuptake correlations with the cognitive tasks were located predominantly in the bilateral temporoparietal cortex and left cuneus/ PCC. Category Fluency was more extensively correlated to FDG uptake than ACE-R Memory. The regional distribution of reduced FDG uptake in the temporoparietal neocortex was clearly distinct from the MR imaging results, in which correla- tions were mainly found in regions within the extended Papez circuit (HCMP, anterior temporal lobe, medial orbitofrontal cortex; Fig 2).

\section{Analysis of Specific Neocortical Nodes: Region-of-Interest Results}

A region-of-interest analysis was performed to directly measure GM volume and FDG uptake in 14 bilateral cortical regions, independent of cognition, in the 3 diagnostic groups. In general, the region-ofinterest volume analysis (Table 3 ) closely reflected the morphologic changes assessed by voxelwise regression analysis. The strongest regional effects observed in the MTL included the bilateral HCMP and bilateral parahippocampal gyrus. Other temporal regions affected were the bilateral medial anterior temporal lobe and the left posterior temporal lobe. There was also a significant group effect of volume in the left middle frontal and left superior parietal lobe. Repeated-measures ANOVA demonstrated a significant lateralized and regionally specific effect of diagnosis $(P=.045$ for the interaction between diagnosis and hemisphere and $P=.016$ among diagnosis, hemisphere, and regions).

With respect to FDG uptake (Table 4), significant and regionally different group effects were also observed in the temporal lobes, in- 


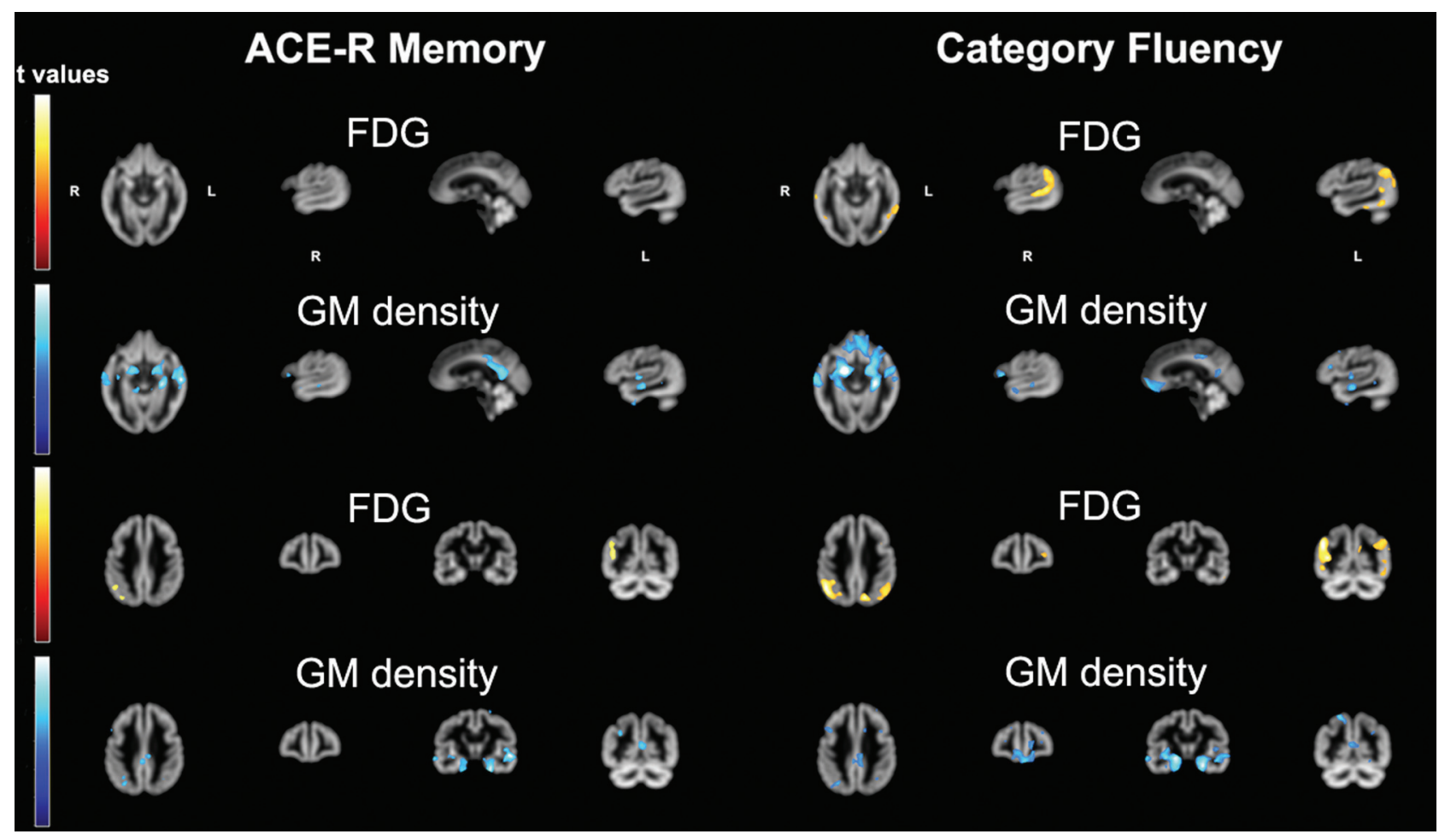

FIG 2. The SPM5 voxelwise regression results for each cognitive task ( $P<.05$, corrected for multiple comparisons). Color bars represent $t \mathrm{values}$ $(\min 0-\max \geqq 6)$.

cluding the left HCMP, left parahippocampal cortex, and left lateral anterior temporal lobe. A group effect was also present in the left PCC and left medial orbitofrontal cortex and bilaterally in the middle frontal cortex, superior parietal, cortex, inferolateral parietal, and thalamus. Repeated-measures ANOVA demonstrated significant mean and regionally different effects of diagnosis on FDG uptake ( $P=.049$ for main effect, $P=.010$ for interaction), while changes were less strongly lateralized than for regional GM volume $(P=.063$ for interaction with regions and hemisphere).

\section{Analysis of WM Fiber Tracts}

The TBSS maps (Fig 3) demonstrate widespread decreases of FA in the major association tracts in $\mathrm{MCI}$, and even more significantly in $\mathrm{AD}$. The cingulum, uncinate, and superior longitudinal fasciculus white matter pathways had clear significant decreases of FA in patients with both $\mathrm{MCI}$ and $\mathrm{AD}$, relative to the healthy controls.

The regional investigation by using the individual Johns Hopkins University tract atlases (Table 5, http://cmrm.med.jhmi.edu/) demonstrated that the effect of diagnostic groups was largely lateralized to the left hemisphere in WM tracts, including the cingulum bundle (hippocampal and cingulate gyrus portion), uncinate fasciculus, inferior fronto-occipital fasciculus, and the superior longitudinal fasciculus. The anterior thalamic radiation in the right hemisphere demonstrated a group effect. Both the forceps major and forceps minor revealed a group effect.

\section{Analysis of the Relationship among FA, Neocortical Nodes, and Cognition}

To investigate the relationship among WM pathways, neocortical nodes, and cognition, we performed a number of Pearson correlations and multiple regression analyses. These analyses were re- stricted to the left hemisphere regions because across the 3 imaging modalities, the left hemisphere had demonstrated the most prominent and significant abnormality in patients with AD.

First the FA within a WM tract of interest was entered into a correlation matrix along with a pair of neocortical nodes that the WM tract connected; the correlation between FA and cognition was also tested (ACE-R Memory and Category Fluency; ACE-R Attention and Orientation was not tested because it revealed no significant correlations with either GM or FDG uptake in the SPM5 analyses). A backward stepwise regression was then performed with a cognitive test as the dependent variable. This analysis determined which aspects of the network under investigation predicted cognition in the most significant and parsimonious regression model (Table 6 summarizes the correlation and regression analyses; Fig 1 displays the networks investigated). Fractional anisotropy always remained a significant predictor of cognition in each network investigated (except for network 3). Regional network atrophy correlated more with memory performance, whereas reduced FDG uptake was more consistently related to category fluency score.

\section{DISCUSSION}

The current investigation has demonstrated that there is a coherent breakdown of the function and integrity of the extended MTL-memory network across the spectrum of disease severity from healthy controls to patients with AD. Decreases of FA within specific WM fiber tracts are significantly correlated to the features (GM volume and FDG uptake) of the neocortical nodes at either end of the tract and to cognition in a network-dependent manner. A salient secondary finding of the investigation is that within this 


\begin{tabular}{|c|c|c|c|c|c|c|c|c|}
\hline \multirow[b]{3}{*}{ Region } & \multicolumn{8}{|c|}{ GM Volume (\% of TIV) } \\
\hline & \multicolumn{2}{|c|}{$\mathrm{HC}$} & \multicolumn{2}{|c|}{$\mathrm{MCl}$} & \multicolumn{2}{|c|}{$A D$} & \multirow[b]{2}{*}{$F 2,27$} & \multirow[b]{2}{*}{ Sig. } \\
\hline & Mean & SD & Mean & SD & Mean & SD & & \\
\hline \multicolumn{9}{|l|}{ Temporal lobe } \\
\hline Hippocampus R & $.120^{\mathrm{b}, \mathrm{d}}$ & .004 & $.104^{\mathrm{c}, \mathrm{d}}$ & .016 & $.099^{\mathrm{b}, \mathrm{c}}$ & .010 & 8.3 & $<.005$ \\
\hline Hippocampus L & $.105^{\mathrm{d}}$ & .005 & $.096^{\mathrm{d}}$ & .016 & $.075^{b, c}$ & .012 & 15.3 & $<.001$ \\
\hline Parahippocampal and ambient gyri $\mathrm{R}$ & $.173^{\mathrm{d}}$ & .012 & .168 & .018 & $.151^{\mathrm{c}}$ & .013 & 5.7 & $<.01$ \\
\hline Parahippocampal and ambient gyri L & $.180^{\mathrm{d}}$ & .008 & .173 & .022 & $.158^{\mathrm{c}}$ & .015 & 4.2 & $<.05$ \\
\hline Anterior TL lateral part R & .135 & .018 & .123 & .018 & .119 & .017 & 2.2 & NS \\
\hline Anterior TL lateral part L & .118 & .015 & .111 & .016 & .101 & .016 & 2.8 & NS \\
\hline Anterior TL medial part R & $.272^{d}$ & .022 & .251 & .025 & $.227^{c}$ & .035 & 6.6 & $<.01$ \\
\hline Anterior TL medial part L & .254 & .028 & .241 & .029 & .210 & .046 & 3.9 & $<.05$ \\
\hline Posterior TL R & 1.35 & .086 & 1.32 & .115 & 1.25 & .096 & 2.5 & NS \\
\hline Posterior TL L & $1.32^{\mathrm{d}}$ & .086 & 1.26 & .136 & $1.18^{c}$ & .119 & 3.4 & $<.05$ \\
\hline \multicolumn{9}{|l|}{ Insula and cingulate gyri } \\
\hline Insula R & $.414^{\mathrm{d}}$ & .025 & .378 & .048 & $.365^{c}$ & .029 & 5.0 & $<.05$ \\
\hline Insula L & $.432^{\mathrm{d}}$ & .030 & .395 & .057 & $.370^{c}$ & .031 & 5.3 & $<.05$ \\
\hline Anterior cingulate $\mathrm{R}$ & .272 & .027 & .270 & .030 & .282 & .019 & .49 & NS \\
\hline Anterior cingulate $\mathrm{L}$ & .282 & .033 & .272 & .029 & .274 & .020 & .42 & NS \\
\hline Posterior cingulate $\mathrm{R}$ & .261 & .029 & .264 & .017 & .266 & .023 & .09 & NS \\
\hline Posterior cingulate L & .273 & .028 & .270 & .024 & .275 & .021 & .12 & NS \\
\hline \multicolumn{9}{|l|}{ Frontal lobe } \\
\hline Middle frontal gyrus R & 1.19 & .065 & 1.13 & .089 & 1.11 & .093 & 2.5 & NS \\
\hline Middle frontal gyrus $L$ & $1.13^{c}$ & .063 & 1.08 & .119 & $1.02^{c}$ & .091 & 3.3 & .05 \\
\hline Medial orbitofrontal R & .184 & .013 & .159 & .042 & .156 & .035 & 2.3 & NS \\
\hline Medial orbitofrontal L & .195 & .014 & .181 & .031 & .166 & .036 & 2.6 & NS \\
\hline Subgenual anterior cingulate $R$ & .039 & .005 & .036 & .015 & .046 & .016 & .01 & NS \\
\hline Subgenual anterior cingulate $\mathrm{L}$ & .046 & .006 & .046 & .009 & .046 & .016 & .24 & NS \\
\hline \multicolumn{9}{|l|}{ Parietal/occipital lobe } \\
\hline Cuneus R & .271 & .031 & .246 & .051 & .243 & .064 & 1.0 & NS \\
\hline Cuneus L & .292 & .029 & .273 & .043 & .257 & .063 & 1.4 & NS \\
\hline Superior parietal lobe $\mathrm{R}$ & 1.04 & .111 & .866 & .265 & .853 & .217 & 2.4 & NS \\
\hline Superior parietal lobe $\mathrm{L}$ & 1.02 & .089 & .977 & .095 & .847 & .211 & 4.2 & $<.05$ \\
\hline Inferolateral parietal lobe R & 1.07 & .099 & 1.04 & .098 & 1.01 & .079 & 1.1 & NS \\
\hline Inferolateral parietal lobe L & 1.07 & .086 & 1.04 & .114 & .968 & .080 & 2.6 & NS \\
\hline \multicolumn{9}{|l|}{ Central structures } \\
\hline Thalamus R & .186 & .019 & .177 & .024 & .180 & .024 & .50 & NS \\
\hline Thalamus $\mathrm{L}$ & .199 & .026 & .199 & .026 & .194 & .022 & .01 & NS \\
\hline Caudate R & .170 & .019 & .160 & .021 & .161 & .020 & .86 & NS \\
\hline Caudate L & .160 & .029 & .157 & .026 & .153 & .015 & .16 & NS \\
\hline Putamen $\mathrm{R}$ & .155 & .019 & .146 & .030 & .147 & .025 & .41 & NS \\
\hline Putamen L & .167 & .019 & .160 & .028 & .162 & .022 & .29 & NS \\
\hline
\end{tabular}

Note:- -HC indicates healthy controls; NS, not significant; Sig., Significance; R, right; L, left; TL, temporal lobe; TIV, total intracranial volume.

a Superscript letters indicate significant pair-wise comparisons revealed by post hoc testing (Tamhane T2, $P=.05$ ).

b Significantly different from $\mathrm{MCl}$.

'Significantly different from HC.

d Significantly different from AD.

old-age memory clinic population, GM atrophy of the extended MTL network was the most prominent phenomenon in patients with sporadic late-onset $\mathrm{AD}$.

The most prominent cognitive impairments in the patients with MCI and AD were in episodic memory and semantic cognition (Table 2). There is evidence from previous studies that this pattern of cognitive deterioration is typical in MCI and late-onset $\mathrm{AD}$ (see Carter et $\mathrm{al}^{20}$ and Wilson et $\mathrm{al}^{21}$ for neuropsychological analyses). The MTL, particularly the HCMP, is integral for episodic memory function. ${ }^{3,4}$ Semantic cognition is dependent on several different regions that include the anterolateral temporal, frontal, and temporoparietal regions. ${ }^{36,37}$ As expected, memory performance was most strongly associated with MTL atrophy, whereas semantic performance had a more distributed correlation with GM atrophy in the anterior temporal lobe and medial frontal regions. These regions form part of a distributed semantic network. ${ }^{22,36,37}$

In addition to the temporofrontal pattern of GM atrophy, predominant reductions in glucose metabolism existed in the posterior temporal and inferolateral parietal cortices, which had a strong correlation to semantic cognition. These data support the view that the semantic impairment and the associated reduction of FDG uptake are secondary to the leading neurodegeneration within the Papez circuit and may ultimately be the consequence of disconnection from the MTL regions. ${ }^{18,38-40}$ The observation that widespread atrophy did not correspond well to reduced FDG uptake in the temporoparietal regions is consistent with the concept of functional disconnection of those regions.

The TBSS data clearly demonstrated that there was a significant reduction of FA throughout the brains of patients with both 


\begin{tabular}{|c|c|c|c|c|c|c|c|c|}
\hline \multirow[b]{3}{*}{ Region } & \multicolumn{8}{|c|}{ FDG Uptake Ratio } \\
\hline & \multicolumn{2}{|c|}{$\mathrm{HC}$} & \multicolumn{2}{|c|}{$\mathrm{MCl}$} & \multicolumn{2}{|c|}{$A D$} & \multirow[b]{2}{*}{$F 2,27$} & \multirow[b]{2}{*}{ Sig. } \\
\hline & Mean & SD & Mean & SD & Mean & SD & & \\
\hline \multicolumn{9}{|l|}{ Temporal lobe } \\
\hline Hippocampus R & .615 & .036 & .576 & .069 & .583 & .024 & 1.9 & NS \\
\hline Hippocampus L & $.619^{\mathrm{b}}$ & .030 & .573 & .069 & $.517^{\mathrm{c}}$ & .076 & 6.6 & $<.01$ \\
\hline Parahippocampal and ambient gyri $\mathrm{R}$ & .597 & .052 & .569 & .055 & .580 & .036 & .90 & NS \\
\hline Parahippocampal and ambient gyri L & .612 & .044 & .576 & .038 & .545 & .067 & 4.5 & $<.05$ \\
\hline Anterior TL lateral part $\mathrm{R}$ & .692 & .064 & .639 & .064 & .672 & .051 & 2.1 & NS \\
\hline Anterior TL lateral part L & $.733^{\mathrm{b}}$ & .052 & $.672^{c}$ & .062 & .661 & .063 & 4.4 & $<.05$ \\
\hline Anterior TL medial part R & .564 & .052 & .532 & .065 & .572 & .049 & 1.4 & NS \\
\hline Anterior TL medial part L & .590 & .033 & .539 & .055 & .557 & .063 & 2.9 & NS \\
\hline Posterior TL R & .853 & .048 & .826 & .049 & .814 & .058 & 1.5 & NS \\
\hline Posterior TL L & $.875^{\mathrm{b}}$ & .048 & .831 & .068 & $.789^{c}$ & .078 & 4.2 & $<.05$ \\
\hline \multicolumn{9}{|l|}{ Insula and cingulate gyri } \\
\hline Insula R & .799 & .040 & .766 & .048 & .773 & .030 & 1.9 & NS \\
\hline Insula L & .789 & .048 & .767 & .049 & .764 & .043 & .82 & NS \\
\hline Anterior cingulate $\mathrm{R}$ & .865 & .039 & .850 & .051 & .886 & .026 & 1.8 & NS \\
\hline Anterior cingulate $\mathrm{L}$ & .867 & .034 & .843 & .051 & .869 & .030 & 1.3 & NS \\
\hline Posterior cingulate $\mathrm{R}$ & 1.06 & .048 & 1.03 & .074 & 1.01 & .027 & 2.2 & NS \\
\hline Posterior cingulate L & $1.05^{\mathrm{b}}$ & .047 & 1.01 & .068 & $.969^{c}$ & .038 & 5.9 & $<.01$ \\
\hline \multicolumn{9}{|l|}{ Frontal lobe } \\
\hline Middle frontal gyrus $\mathrm{R}$ & $1.07^{\mathrm{b}, \mathrm{d}}$ & .060 & $.993^{\mathrm{c}}$ & .074 & $.988^{c}$ & .044 & 5.9 & $<.01$ \\
\hline Middle frontal gyrus L & $1.07^{\mathrm{b}}$ & .048 & .999 & .073 & $.983^{c}$ & .048 & 5.6 & $<.01$ \\
\hline Medial orbitofrontal R & .865 & .033 & .832 & .075 & .859 & .032 & 1.2 & NS \\
\hline Medial orbitofrontal L & .854 & .032 & .798 & .064 & .827 & .032 & 4.0 & $<.05$ \\
\hline Subgenual anterior cingulate $\mathrm{R}$ & .654 & .041 & 631 & .054 & .660 & .065 & .84 & NS \\
\hline Subgenual anterior cingulate $\mathrm{L}$ & .665 & .044 & .623 & .079 & .665 & .073 & 1.4 & NS \\
\hline \multicolumn{9}{|l|}{ Parietal/occipital lobe } \\
\hline Cuneus R & .991 & .058 & .963 & .089 & .946 & .083 & .84 & NS \\
\hline Cuneus L & .991 & .049 & .994 & .065 & .925 & .114 & 2.3 & NS \\
\hline Superior parietal lobe $\mathrm{R}$ & $1.02^{b, d}$ & .033 & $.956^{\mathrm{c}}$ & .051 & $.933^{c}$ & .041 & 11.3 & $<.001$ \\
\hline Superior parietal lobe L & $1.02^{\mathrm{b}}$ & .038 & .974 & .067 & $.913^{c}$ & .057 & 7.9 & $<.01$ \\
\hline Inferolateral parietal lobe $\mathrm{R}$ & $1.02^{\mathrm{b}}$ & .057 & .962 & .069 & $.918^{\mathrm{c}}$ & .061 & 6.2 & $<.01$ \\
\hline Inferolateral parietal lobe L & $1.02^{\mathrm{b}}$ & .036 & .945 & .083 & $.884^{\mathrm{c}}$ & .079 & 8.8 & .001 \\
\hline \multicolumn{9}{|l|}{ Central structures } \\
\hline Thalamus $\mathrm{R}$ & $.849^{\mathrm{b}}$ & .063 & .791 & .088 & $.760^{c}$ & .035 & 4.2 & $<.05$ \\
\hline Thalamus L & $.846^{\mathrm{b}}$ & .060 & .808 & .087 & $.762^{c}$ & .051 & 3.4 & .05 \\
\hline Caudate R & .851 & .088 & .799 & .143 & .795 & .067 & .86 & NS \\
\hline Caudate L & .844 & .104 & .817 & .104 & .785 & .040 & .97 & NS \\
\hline Putamen R & 1.06 & .063 & 1.08 & .094 & 1.10 & .074 & .75 & NS \\
\hline Putamen L & 1.06 & .062 & 1.08 & .085 & 1.08 & .059 & .41 & NS \\
\hline
\end{tabular}

Note:-HC indicates healthy controls; NS, not significant; Sig., Significance; R, right; L, left; TL, temporal load.

${ }^{a}$ Superscript letters indicate significant pair-wise comparisons revealed by post hoc testing (Tamhane T2, $P=.05$ ).

b Significantly different from AD.

c Significantly different from HC.

d Significantly different from $\mathrm{MCl}$.

$\mathrm{MCI}$ and $\mathrm{AD}$ in most major WM fiber tracts relative to the healthy controls (Fig 3), similar to that in a previous study. ${ }^{17}$ The regional FA data obtained with the Johns Hopkins University tract atlas supplemented the voxelwise TBSS findings, particularly in the left hemisphere, where significant FA reductions were found in the anterior cingulum (cingulate gyrus portion), posterior cingulum (hippocampal portion), uncinate fasciculus, inferior fronto-occipital fasciculus, and superior longitudinal fasciculus (containing the arcuate fasciculus). The left hemisphere bias of reduced FA in Alzheimer disease has been reported in other studies. ${ }^{40,41}$

Relating reduced FA in specific white matter tracts with regional cortical atrophy and reduced FDG uptake allowed testing of the hypothesis that the structural changes and functional regional network changes are closely related to each other and to cognitive impairment. Of the 6 networks that were investigated, only 1 (network 3 ) revealed no significant correlation between FA and either cognitive domain; this was the anterior portion of the cingulum bundle. At the posterior cingulum and uncinate fasciculus, the data indicate that neurodegeneration at the anterior temporal lobe and hippocampus was correlated to both reduced FA within the tracts and reduced FDG uptake in the neocortical node at the projection site (the PCC and medial orbitofrontal cortex, respectively). One of the most pertinent findings from the regression analyses was that for each network (except network 3), FA within the tract always remained as a significant predictor of cognition in the most significant and parsimonious regression model. Our findings are consistent with previously published reports ${ }^{17-19}$ that demonstrated that patients with MCI and AD show reduced FA in the WM to a similar extent, ${ }^{17}$ that microstructure (FA) and function (hypometabolism) are correlated to cognition, ${ }^{19}$ and that primary GM lesions lead to disrupted WM and hypometabolism. ${ }^{17,18}$ 
AD

$\mathbf{R}$

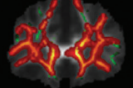

$\mathbf{L}$
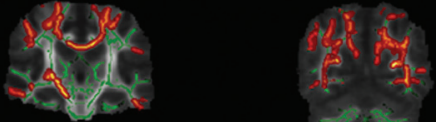

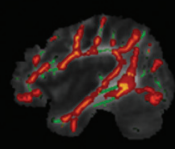

$\mathbf{R}$

$\mathbf{R}$
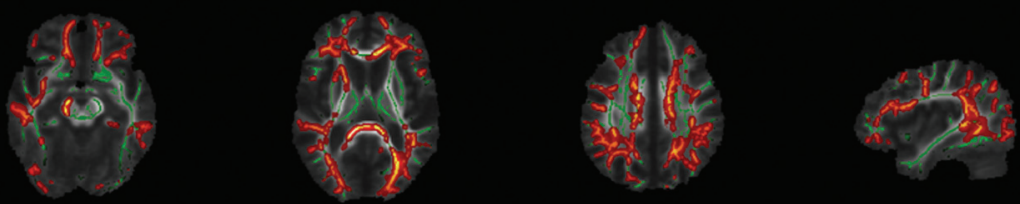

$\mathbf{L}$

$\mathbf{L}$

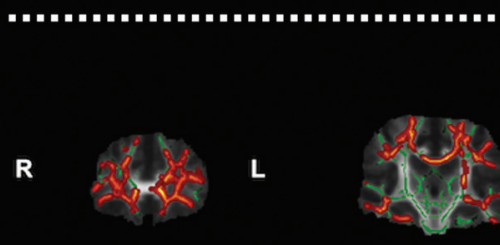

MCI

$\mathbf{R}$

$\mathbf{R}$
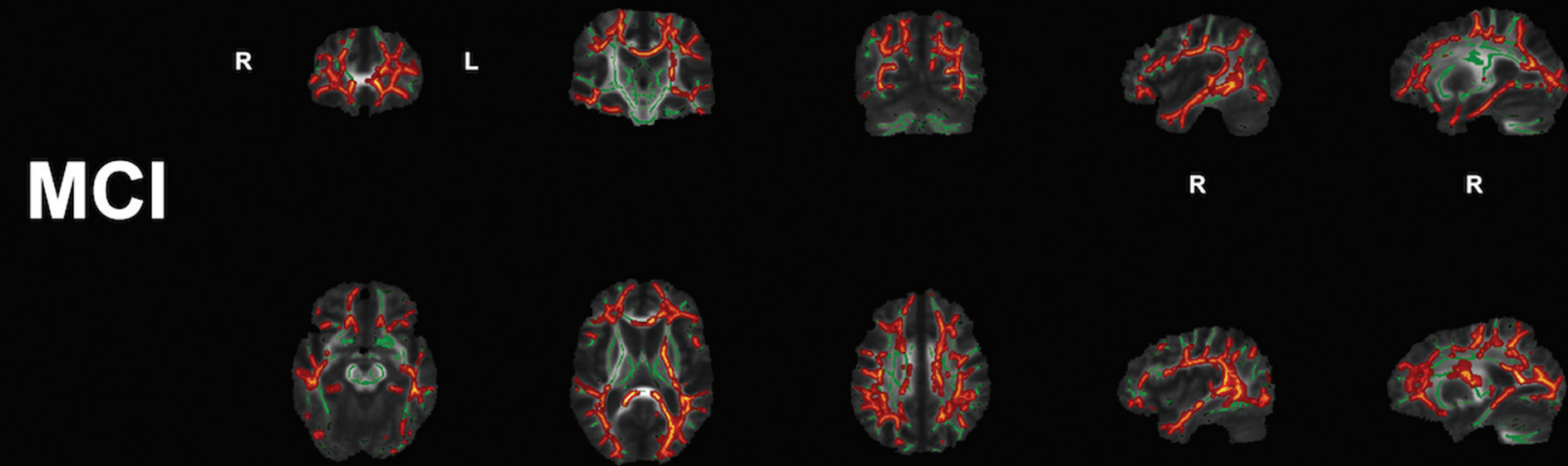

$=$
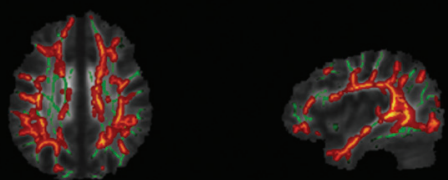

L

$\mathbf{L}$

FIG 3. Abnormal ( $P<.05$, compared with controls) FA depicted by the hot-body color scale with a TBSS tract skeleton (green) in the background.

Table 5: Regional analyses of FA values within WM tracts ${ }^{\mathrm{a}}$

\begin{tabular}{|c|c|c|c|c|c|c|c|c|}
\hline \multirow[b]{3}{*}{ Fibre Tract } & \multicolumn{8}{|c|}{ WM Tract FA Values } \\
\hline & \multicolumn{2}{|c|}{$\mathrm{HC}$} & \multicolumn{2}{|c|}{$\mathrm{MCl}$} & \multicolumn{2}{|c|}{$A D$} & \multirow[b]{2}{*}{$F(2,27)$} & \multirow[b]{2}{*}{ Sig. } \\
\hline & Mean & SD & Mean & SD & Mean & SD & & \\
\hline Anterior thalamic radiation $\mathrm{R}$ & $.359^{\mathrm{b}}$ & .375 & .334 & .039 & $.313^{c}$ & .036 & 4.42 & $<.05$ \\
\hline Anterior thalamic radiation $\mathrm{L}$ & .375 & .026 & .356 & .036 & .340 & .039 & 2.80 & NS \\
\hline Cingulum (cingulate gyrus portion) $\mathrm{R}$ & .427 & .034 & .399 & .053 & .395 & .052 & 1.33 & NS \\
\hline Cingulum (cingulate gyrus portion) L & .502 & .030 & .458 & .057 & .458 & .045 & 3.27 & .05 \\
\hline Cingulum (hippocampal portion) R & .357 & .028 & .324 & .064 & .314 & .038 & 2.29 & NS \\
\hline Cingulum (hippocampal portion) L & $.375^{\mathrm{b}}$ & .037 & .338 & .049 & $.299^{c}$ & .029 & 8.06 & $<.01$ \\
\hline Uncinate fasciculus $\mathrm{R}$ & .376 & .034 & .366 & .042 & .335 & .034 & 2.89 & NS \\
\hline Uncinate fasciculus $L$ & $.375^{\mathrm{b}}$ & .027 & .352 & .053 & $.325^{c}$ & .034 & 3.74 & $<.05$ \\
\hline Inferior fronto-occipital fasciculus $\mathrm{R}$ & .399 & .017 & .379 & .048 & .371 & .037 & 1.67 & NS \\
\hline Inferior fronto-occipital fasciculus $L$ & $.397^{\mathrm{b}}$ & .023 & .371 & .038 & $.362^{c}$ & .027 & 3.67 & $<.05$ \\
\hline Inferior longitudinal fasciculus R & .418 & .019 & .375 & .059 & .382 & .051 & 2.73 & NS \\
\hline Inferior longitudinal fasciculus L & .389 & .019 & .361 & .035 & .365 & .049 & 2.10 & NS \\
\hline Superior longitudinal fasciculus $\mathrm{R}$ & .369 & .021 & .341 & .043 & .355 & .032 & 1.99 & NS \\
\hline Superior longitudinal fasciculus $L$ & $.375^{\mathrm{b}}$ & .022 & $.340^{c}$ & .037 & .342 & .038 & 3.90 & $<.05$ \\
\hline Forceps major & $.528^{\mathrm{b}}$ & .041 & .498 & .059 & $.466^{c}$ & .040 & 3.79 & $<.05$ \\
\hline Forceps minor & $.394^{\mathrm{b}, \mathrm{d}}$ & .025 & $.357^{c}$ & .037 & $.343^{c}$ & .029 & 7.22 & $<.01$ \\
\hline Corticospinal tract $\mathrm{R}$ & .533 & .025 & .498 & .061 & .504 & .065 & 1.38 & NS \\
\hline Corticospinal tract $\mathrm{L}$ & .525 & .026 & .493 & .047 & .500 & .039 & 2.05 & NS \\
\hline
\end{tabular}

Note:-HC indicates healthy controls; NS, not significant; Sig., Significance; R, right; L, left.

a Superscript letters indicate significant pair-wise comparisons revealed by post hoc testing (Tamhane T2, $P=.05$ ).

b Significantly different from AD.

c Significantly different from $\mathrm{HC}$.

${ }^{\mathrm{d}}$ Significantly different from MCl. 


\begin{tabular}{|c|c|c|c|}
\hline \multirow[b]{2}{*}{$\begin{array}{l}\text { White Matter Tract and Associated } \\
\text { Neocortical Nodes }\end{array}$} & \multirow[b]{2}{*}{$\begin{array}{l}\text { Correlation with } \\
\text { Tract FA }\end{array}$} & \multicolumn{2}{|c|}{ Multiple Regression Results } \\
\hline & & Relation with ACE-R-Memory & $\begin{array}{c}\text { Relation with Total Category } \\
\text { Fluency Score }\end{array}$ \\
\hline Network 1 & & $R^{2}=.60 ; F 2,27=20.0 ; P<.001$ & $R^{2}=.67 ; F 2,27=27.4 ; P<.001$ \\
\hline Cingulum (hippocampal portion) FA & & $b=.29, P=.05$ & $b=.37, P<.01$ \\
\hline Hippocampal volume & $r=.54, P<.01$ & $b=.58, P<.001$ & $b=.56, P<.001$ \\
\hline Hippocampal FDG & $r=.47, P<.01$ & NS & NS \\
\hline PCC volume & NS & NS & NS \\
\hline PCC FDG & $r=.55, P<.01$ & NS & NS \\
\hline Network 2 & & $R^{2}=.41 ; \mathrm{F} 2,27=9.34 ; P<.001$ & $R^{2}=.47 ; \mathrm{F} 2,27=12.1 ; P<.001$ \\
\hline Uncinate fasciculus FA & & $b=.39, P<.01$ & $b=.37, P<.05$ \\
\hline Medial ATL volume & $r=.40, P<.05$ & NS & NS \\
\hline Medial ATL FDG & NS & NS & NS \\
\hline Medial orbital frontal volume & NS & $b=.42, P=.01$ & $b=.49, P<.01$ \\
\hline Medial orbital frontal FDG & $r=.60, P<.01$ & NS & NS \\
\hline Network 3 & & $R^{2}=.20 ; \mathrm{F} 2,27=7.1 ; P<.05$ & $R^{2}=.20 ; \mathrm{F} 2,27=6.9 ; P<.05$ \\
\hline Cingulum (cingulate portion) FA & & NS & NS \\
\hline PCC volume & NS & NS & NS \\
\hline PCC FDG & $r=.39, P<.05$ & $b=.45, P<.05$ & $b=.45, P<.05$ \\
\hline ACC volume & NS & NS & NS \\
\hline ACC FDG & NS & NS & NS \\
\hline Network 4 & & $R^{2}=.62 ; \mathrm{F} 2,27=15.2 ; P<.001$ & $R^{2}=.53 ; \mathrm{F} 2,27=15.2 ; P<.001$ \\
\hline Superior longitudinal fasciculus FA & & $b=.51, P=.001$ & $b=.29, P<.05$ \\
\hline Inferolateral parietal volume & NS & $b=.73, P=.001$ & NS \\
\hline Inferolateral parietal FDG & NS & $b=.47, P<.01$ & $b=.63, P<.001$ \\
\hline Middle frontal volume & NS & NS & NS \\
\hline Middle frontal FDG & NS & $b=-.59, P<.05$ & NS \\
\hline Network 5 & & $R^{2}=.54 ; \mathrm{F} 2,27=11.8 ; P<.001$ & $R^{2}=.54 ; \mathrm{F} 2,27=10.2 ; P<.001$ \\
\hline Superior longitudinal fasciculus FA & & $b=.31, P<.05$ & $b=.27, P=.06$ \\
\hline Superior parietal volume & NS & $b=.48, P=.001$ & $b=.37, P<.05$ \\
\hline Superior parietal FDG & NS & $b=.31, P<.05$ & $b=.44, P<.01$ \\
\hline Middle frontal volume & NS & NS & NS \\
\hline Middle frontal FDG & NS & NS & NS \\
\hline Network 6 & & $R^{2}=.44 ; \mathrm{F} 2,27=10.8 ; P<.001$ & $R^{2}=.52 ; \mathrm{F} 2,27=9.5 ; P<.001$ \\
\hline Inferior fronto-occipital fasciculus FA & & $b=.40, P<.05$ & $b=.27, P=.08$ \\
\hline Posterior temporal volume & NS & NS & $b=.36, P<.05$ \\
\hline Posterior temporal FDG & NS & NS & $b=.31, P=.07$ \\
\hline Middle frontal volume & NS & $b=.47, P<.01$ & NS \\
\hline Middle frontal FDG & $r=.52, P<.01$ & NS & NS \\
\hline
\end{tabular}

Note:-NS indicates not significant; ATL, anterior temporal lobe.

The present data do not confirm the hypothesis that synaptic dysfunction, as reflected in glucose hypometabolism, ${ }^{42}$ leads to neuronal loss and tissue atrophy. Atrophy of the MTL was the leading lesion in our study, and structural white matter changes also provided an independent significant contribution to explain the main cognitive deficits in accordance with recent previous studies. ${ }^{17-19}$ The reduced temporoparietal FDG uptake as a marker of synaptic dysfunction may be the consequence of local toxic effects of amyloid deposition, which is known to precede glucose impairment in those regions. ${ }^{43}$ Cortical dysfunction could also be mediated by loss of brain-derived neurotrophic factor secondary to axonal impairment and disconnection, probably compounded by a loss of cholinergic fibers, ${ }^{44}$ which cannot be visualized by current MR imaging techniques but has been indicated by a reduction of acetylcholine esterase activity. ${ }^{45}$

Regional comparisons between the magnitude of atrophy and glucose metabolism raise some technical measurement issues. The presence of hippocampal hypometabolism is contentious, with some authors suggesting relative metabolic preservation ${ }^{46-49}$ and others suggesting metabolic deterioration. ${ }^{38,50-54}$ A possible explanation for the discrepancy in the current investigation is likely to be caused by 3 methodologically related phenomena: PET scanner resolution, partial volume correction, and smoothing of image data. Currently available algorithms for partial volume correction depend critically on the accuracy of image registration and segmentation and are based on assumptions about regional homogeneity that may not actually be fulfilled. ${ }^{55,56} \mathrm{We}$, therefore, did not include partial volume correction but took advantage of the isotropic spatial resolution of $2.5 \mathrm{~mm}$ of the highresolution research tomograph scanner, while previous investigations used PET scanners with a spatial resolution of $\geq 5 \mathrm{~mm}$. To preserve as much of this advantage as possible, we also used advanced spatial normalization procedures and a highly accurate atlas for region-of-interest analysis and spatial filtering by only 8 $\mathrm{mm}$ for voxelwise comparisons.

There is still a difference in the intrinsic spatial resolution between PET and MR imaging that may limit the detection of metabolic changes in small structures such as the HCMP when using PET and thus may have influenced the results. While these issues cannot currently be resolved completely, both the SPM5 regression and region-of-interest analyses clearly show that MTL atrophy was the most significant phenomenon. Even in the regions 
where reduced FDG uptake predominated, this was not in the complete absence of atrophy, suggesting that at least part of the reduction in FDG uptake could be explained by the partial volume effect.

There is a possibility that regional atrophy may influence the FA data; however, atrophy would not completely explain the data for at least 2 reasons: First, both methods of testing FA (regionof-interest and TBSS) broadly support each other; namely that there are FA reductions in patients with both MCI and AD. This feature is important because the TBSS analysis is performed in a template space derived from the study population and only the voxels with the highest FA value perpendicular to the tract skeleton (again derived from the study population) are included in the voxelwise analysis. This inclusion protects against slight warping/ registration errors and ensures that the actual WM tract of interest is sampled or not the GM of the CSF, for example. Second, the region-of-interest analysis, which relies on estimating the inverse warps, taking the Johns Hopkins University atlases back into native diffusion space, revealed results similar to those of the TBSS analysis, particularly for the left hemisphere.

There are several limitations that affect the current investigation. First, because only a small number of participants were investigated, there is limited statistical power. Additionally, a large number of statistical comparisons were performed, particularly for the region-of-interest analyses (Tables 3-5), without multiple-comparison correction. However, the main regional effects were also confirmed by SPM5 analysis, which was corrected for multiple comparisons; and after each stage of analysis, subsequent tests were restricted to those regions with the most significant group effects in the previous stages of the analysis. For instance, only the neuropsychological tests with the most significant group effects were used (ACE-R Memory and Category Fluency), and only regions within networks in the left hemisphere were tested (because there was a significant lateralized effect of GM atrophy). Another limitation is caused by the fact that healthy controls were significantly younger than the patients with $\mathrm{AD}$; though this difference had no significant effect on the neuropsychology data, it may have contributed to the imaging findings and was taken into account as a confounding variable. A final consideration is that the current data were cross-sectional, and as such, no firm conclusions can be made about the causal nature of primary lesions resulting in impairment of FA and neocortical hypometabolism. Longitudinal follow-up would be required to verify this hypothesis.

\section{CONCLUSIONS}

Combining multitechnique neuroimaging data has demonstrated that the cognitive network failure in $\mathrm{MCI}$ and $\mathrm{AD}$ is characterized by multiple lesions of network components within the Papez circuit and extended neocortical association areas. Atrophy (HCMP/anterior temporal lobe) was a prominent feature that was correlated to FA in the fasciculi of interest (cingulum/uncinate), which was also correlated to hypometabolism in projection sites (PCC, medial orbitofrontal cortex). These data suggest a close association between regional neuronal loss and impaired WM integrity, leading to neocortical disconnection, resultant hypometabolism, and impairment of cognition.

\section{ACKNOWLEDGMENTS}

We are especially grateful to the patients and their caregivers for taking part in this investigation.

Disclosures: Alistair Burns-UNRELATED: Expert Testimony: occasional court report, Royalties: Editor-in-Chief of International Journal of Geriatric Psychiatry. Matthew A. Lambon Ralph—UNRELATED: Board Membership: Medical Research Council (UK), Comments: nonclinical fellowship panel, Grants/Grants Pending: Medical Research Council programme grant.* Karl Herholz—RELATED: Grant: Medical Research Council,* UNRELATED: Consultancy: GE Healthcare, Eli Lilly, Grants/Grants Pending: European commission,* Alzheimer's Research UK,* AVID radiopharmaceuticals.* *Money paid to the institution.

\section{REFERENCES}

1. Papez JW. A proposed mechanism of emotion. Archives of Neurology and Psychiatry 1937;38:725-43

2. Maclean PD. Some psychiatric implications of physiological studies on frontotemporal portion of limbic system (visceral brain). Electroencephalogr Clin Neurophysiol 1952;4:407-18

3. Scoville WB, Milner B. Loss of recent memory after bilateral hippocampal lesions. J Neurol Neurosurg Psychiatry 1957;20:11-21

4. Aggleton JP, Brown MW. Episodic memory, amnesia, and the hippocampal-anterior thalamic axis. Behav Brain Sci 1999;22:425-44, discussion 444-89

5. Harvey DY, Wei T, Ellmore TM, et al. Neuropsychological evidence for the functional role of the uncinate fasciculus in semantic control. Neuropsychologia 2013;8:00042-40

6. Reijmer YD, Leemans A, Caeyenberghs K, et al. Disruption of cerebral networks and cognitive impairment in Alzheimer disease. Neurology 2013;13:13

7. Zhou J, Greicius MD, Gennatas ED, et al. Divergent network connectivity changes in behavioural variant frontotemporal dementia and Alzheimer's disease. Brain 2010;133:1352-67

8. Braak H, Braak E. Neuropathological stageing of Alzheimer-related changes. Acta Neuropathol 1991;82:239-59

9. Braak H, Braak E. Frequency of stages of Alzheimer-related lesions in different age categories. Neurobiol Aging 1997;18:351-57

10. Thal DR, Rub U, Orantes M, et al. Phases of A beta-deposition in the human brain and its relevance for the development of AD. Neurology 2002;58:1791-800

11. Thal DR, Rub U, Schultz C, et al. Sequence of Abeta-protein deposition in the human medial temporal lobe. J Neuropathol Exp Neurol 2000;59:733-48

12. Fox NC, Cousens $S$, Scahill R, et al. Using serial registered brain magnetic resonance imaging to measure disease progression in Alzheimer disease: power calculations and estimates of sample size to detect treatment effects. Arch Neurol 2000;57:339-44

13. Chua TC, Wen W, Slavin MJ, et al. Diffusion tensor imaging in mild cognitive impairment and Alzheimer's disease: a review. Curr Opin Neurol 2008;21:83-92

14. Frisoni GB, Fox NC, Jack CR Jr, et al. The clinical use of structural MRI in Alzheimer disease. Nat Rev Neurol 2010;6:67-77

15. Herholz K. Cerebral glucose metabolism in preclinical and prodromal Alzheimer's disease. Expert Rev Neurother 2010;10:1667-73

16. Filippi M, Agosta F. Structural and functional network connectivity breakdown in Alzheimer's disease studied with magnetic resonance imaging techniques. J Alzheimers Dis 2011;24:455-74

17. Bozoki AC, Korolev IO, Davis NC, et al. Disruption of limbic white matter pathways in mild cognitive impairment and Alzheimer's disease: a DTI/FDG-PET study. Hum Brain Mapp 2012;33:1792-802

18. Villain N, Fouquet M, Baron JC, et al. Sequential relationships between grey matter and white matter atrophy and brain metabolic abnormalities in early Alzheimer's disease. Brain 2010;133:3301-14

19. Yakushev I, Schreckenberger M, Muller MJ, et al. Functional implications of hippocampal degeneration in early Alzheimer's disease: a combined DTI and PET study. Eur J Nucl Med Mol Imaging 2011;38: 2219-27

20. Carter SF, Caine D, Burns A, et al. Staging of the cognitive decline in 
Alzheimer's disease: insights from a detailed neuropsychological investigation of mild cognitive impairment and mild Alzheimer's disease. Int J Geriatr Psychiatry 2012;27:423-32

21. Wilson RS, Leurgans SE, Boyle PA, et al. Cognitive decline in prodromal Alzheimer disease and mild cognitive impairment. Arch Neurol 2011;68:351-56

22. Acosta-Cabronero J, Patterson K, Fryer TD, et al. Atrophy, hypometabolism and white matter abnormalities in semantic dementia tell a coherent story. Brain 2011;134:2025-35

23. Catani M, Thiebaut de Schotten M. A diffusion tensor imaging tractography atlas for virtual in vivo dissections. Cortex 2008;44: 1105-32

24. van Velden FH, Kloet RW, van Berckel BN, et al. HRRT versus HR+ human brain PET studies: an interscanner test-retest study. J Nucl Med 2009;50:693-702

25. McKhann G, Drachman D, Folstein M, et al. Clinical diagnosis of Alzheimer's disease: report of the NINCDS-ADRDA Work Group under the auspices of Department of Health and Human Services Task Force on Alzheimer's Disease. Neurology 1984;34:939-44

26. Petersen RC. Mild cognitive impairment as a diagnostic entity. J Intern Med 2004;256:183-94

27. Jones DK, Pierpaoli C. Contribution of cardiac pulsation to variability of tractography results. In: Proceedings of the 13th Scientific Meeting of the International Society for Magnetic Resonance in Medicine, Miami Beach, Florida. May 7-13, 2005

28. de Jong H, van Velden FHP, Kloet RW, et al. Performance evaluation of the ECAT HRRT: an LSO-LYSO double layer high resolution, high sensitivity scanner. Phys Med Biol 2007;52:1505-26

29. Wienhard K, Schmand M, Casey ME, et al. The ECAT HRRT: performance and first clinical application of the new high resolution research tomograph. IEEE Trans Nucl Sci 2002;49:104-10

30. Michel C, Schmand M, Liu X, et al. Reconstruction strategies for the HRRT. Nuclear Science Symposium Conference Record 2000 IEEE 2000;2:14/207-15/212

31. Byars LG, Sibomana M, Burbar Z, et al. Variance reduction on randoms from coincidence histograms for the HRRT. Nuclear Science Symposium Conference Record 2005 IEEE 2005;5:2622-26

32. Watson CC. New, faster, image-based scatter correction for 3D PET. Nuclear Science IEEE Transactions on 2000;47:1587-94

33. Sibomana M, Byars L, Panin V, et al. Simultaneous measurement of transmission and emission contamination using a collimated /sup 137/Cs point source for the HRRT. Nuclear Science Symposium Conference Record 2004 IEEE 2004;4:2647-51

34. Embleton KV, Haroon HA, Morris DM, et al. Distortion correction for diffusion-weighted MRI tractography and fMRI in the temporal lobes. Hum Brain Mapp 2010;31:1570-87

35. Smith SM, Nichols TE. Threshold-free cluster enhancement: addressing problems of smoothing, threshold dependence and localisation in cluster inference. Neuroimage 2009;44:83-98

36. Stopford CL, Snowden JS, Thompson JC, et al. Variability in cognitive presentation of Alzheimer's disease. Cortex 2008;44:185-95

37. Visser M, Jefferies E, Lambon Ralph MA. Semantic processing in the anterior temporal lobes: a meta-analysis of the functional neuroimaging literature. J Cogn Neurosci 2010;22:1083-94

38. Chetelat G, Desgranges B, Landeau B, et al. Direct voxel-based comparison between grey matter hypometabolism and atrophy in Alzheimer's disease. Brain 2008;131:60-71

39. Fouquet M, Desgranges B, Landeau B, et al. Longitudinal brain metabolic changes from amnestic mild cognitive impairment to Alzheimer's disease. Brain 2009;132:2058-67

40. Kuczynski B, Targan E, Madison C, et al. White matter integrity and cortical metabolic associations in aging and dementia. Alzheimers Dement 2010;6:54-62

41. Catheline G, Periot O, Amirault M, et al. Distinctive alterations of the cingulum bundle during aging and Alzheimer's disease. Neurobiol Aging 2010;31:1582-92

42. Trojanowski JQ, Vandeerstichele H, Korecka M, et al. Update on the biomarker core of the Alzheimer's Disease Neuroimaging Initiative subjects. Alzheimers Dement 2010;6:230-38

43. Forster S, Grimmer T, Miederer I, et al. Regional expansion of hypometabolism in Alzheimer's disease follows amyloid deposition with temporal delay. Biol Psychiatry 2012;71:792-97

44. Selden NR, Gitelman DR, Salamon-Murayama N, et al. Trajectories of cholinergic pathways within the cerebral hemispheres of the human brain. Brain 1998;121(pt 12):2249-57

45. Haense C, Kalbe E, Herholz K, et al. Cholinergic system function and cognition in mild cognitive impairment. Neurobiol Aging 2012; 33:867-77

46. Desgranges B, Baron JC, de la Sayette V, et al. The neural substrates of memory systems impairment in Alzheimer's disease: a PET study of resting brain glucose utilization. Brain 1998;121(pt 4):611-31

47. Herholz K, Salmon E, Perani D, et al. Discrimination between Alzheimer dementia and controls by automated analysis of multicenter FDG PET. Neuroimage 2002;17:302-16

48. Ishii K, Sasaki M, Yamaji S, et al. Relatively preserved hippocampal glucose metabolism in mild Alzheimer's disease. Dement Geriatr Cogn Disord 1998;9:317-22

49. Minoshima S, Giordani B, Berent S, et al. Metabolic reduction in the posterior cingulate cortex in very early Alzheimer's disease. Ann Neurol 1997;42:85-94

50. De Santi S, de Leon MJ, Rusinek H, et al. Hippocampal formation glucose metabolism and volume losses in MCI and AD. Neurobiol Aging 2001;22:529-39

51. Mosconi L, De Santi S, Li J, et al. Hippocampal hypometabolism predicts cognitive decline from normal aging. Neurobiol Aging 2008;29:676-92

52. Mosconi L, Sorbi S, de Leon MJ, et al. Hypometabolism exceeds atrophy in presymptomatic early-onset familial Alzheimer's disease. J Nucl Med 2006;47:1778-86

53. Mosconi L, Tsui WH, De Santi S, et al. Reduced hippocampal metabolism in MCI and AD: automated FDG-PET image analysis. Neurology 2005;64:1860-67

54. Nestor PJ, Fryer TD, Smielewski P, et al. Limbic hypometabolism in Alzheimer's disease and mild cognitive impairment. Ann Neurol 2003;54:343-51

55. Segobin SH, Matthews JC, Markiewicz PJ, et al. A hybrid between region-based and voxel-based methods for partial volume correction in PET. Nuclear Science Symposium Conference Record (NSS/ MIC) 2010 IEEE 2010;3073-78

56. Gutierrez D, Montandon M-L, Assal F, et al. Anatomically guided voxel-based partial volume effect correction in brain PET: impact of MRI segmentation. Comput Med Imaging Graph 2012;36:610-19

57. Ashburner J, Friston KJ. Unified segmentation. Neuroimage 2005;26:839-51

58. Ashburner J. A fast diffeomorphic image registration algorithm. Neuroimage 2007;38:95-113

59. Smith SM, Jenkinson M, Johansen-Berg H et al. Tract-based spatial statistics: voxelwise analysis of multi-subject diffusion data. Neuroimage 2006;31:1487-1505

60. Smith SM, Johansen-Berg H, Jenkinson $M$ et al. Acquisition and voxelwise analysis of multi-subject diffusion data with tract-based spatial statistics. Nat Protoc 2007;2:499-503

61. Hammers A, Allom R, Koepp MJ et al. Three-dimensional maximum probability atlas of the human brain, with particular reference to the temporal lobe. Hum Brain Mapp 2003;19:224-47

62. Hua K, Zhang J, Wakana S et al. Tract probability maps in stereotaxic spaces: analyses of white matter anatomy and tract-specific quantification. Neuroimage 2008;39:336-47 


\section{APPENDIX}

\section{Materials and Methods}

Participants. There were 32 participants ( 13 healthy controls and 20 patients; 12 patients with MCI and 8 with mild AD) in this investigation, which was approved by the Central Manchester Research Ethics Committee (UK). All patients (and their caregivers/ next of kin) gave signed, informed consent. All patients were recruited from an old-age memory clinic at Wythenshawe Hospital, Manchester, UK. Patients with mild AD were classified as having a Mini-Mental State Examination score typically between 20 and 25 and fulfilled the criteria developed by the National Institute of Neurologic and Communicative Disorders and Stroke-Alzheimer Disease and Related Disorders Association. ${ }^{25}$ Patients with MCI fulfilled the criteria established by Petersen ${ }^{26}$ and were best characterized as amnestic multiple domain; they had Mini-Mental State Examination scores $\geq 26$. Individuals with alcoholism, previous head injury, stroke, epilepsy, other neurologic diseases, or major medical illness (eg, cancer, thyroid dysfunction, anemia, and so forth) were excluded, as were those with an unstable psychiatric illness. Patients underwent brain imaging (CT and electroencephalography) as well as the usual blood screening tests to exclude treatable causes of dementia.

All participants underwent an extensive neuropsychological investigation (a detailed description of the neuropsychological test battery can be found in Carter et $\mathrm{al}^{20}$ ), and all investigations, including neuroimaging, were completed within a 6-week time frame. Head motion was monitored throughout the neuroimaging acquisitions, and participants with excessive head movement were excluded from the study; 3 participants ( 2 healthy controls and 1 patient with $\mathrm{MCI}$ ) were excluded because of excessive head movement. APOE status was determined for most participants (26/30); it was unobtainable for 3 patients with MCI and 1 healthy control. A summary of the basic participant information is found in Table 1. There was a small but significant difference in age among groups, and we, therefore, used age as a covariate in all statistical analyses.

\section{MR Imaging Acquisition}

All MR imaging was performed on the same 3T Achieva scanner (Philips Healthcare) by using an 8-element sensitivity encoding head coil. A T1-weighted inversion recovery acquisition was obtained parallel to the anterior/posterior commissure line with a $3 \mathrm{D}$ acquisition; fast-field echo; field $=256 \times 256$ matrix; sensitivity encoding acceleration factor $=2$; section thickness, $=1$ $\mathrm{mm} ; 150$ contiguous sections; acquired voxel size $=1.0 \times 1.25 \times$ $1.0 \mathrm{~mm}$; reconstructed to $=1.0 \times 1.0 \times 1.0 \mathrm{~mm}$; number of signal averages $=1$; $\mathrm{TR}=8.4 \mathrm{~ms} ; \mathrm{TE}=3.8 \mathrm{~ms}$; $\mathrm{TI}=1150 \mathrm{~ms}$; flip angle $=80^{\circ}$; total scan acquisition $=574$ seconds.

Diffusion-weighted imaging was performed by using a pulsed gradient spin-echo EPI sequence with $\mathrm{TE}=54 \mathrm{~ms}$, TR $=11884$ $\mathrm{ms}, \mathrm{G}$ (phase-encoding gradient) $=62 \mathrm{mTm}^{-1}$, half scan factor $=0.679,112 \times 112$ image matrix reconstructed to $128 \times 128$ by using zero padding, reconstructed resolution $=1.875 \times 1.875$ $\mathrm{mm}$, section thickness $=2.1 \mathrm{~mm}, 60$ contiguous sections, 43 noncollinear diffusion sensitization directions at $b=1200 \mathrm{~s} / \mathrm{mm}^{-2}(\Delta$, $\delta=29.8,13.1 \mathrm{~ms}), 1$ at $b=0$, sensitivity encoding acceleration factor $=2.5$. Each diffusion-weighted volume was acquired entirely before starting on the next diffusion-weighting; this proce- dure resulted in 44 temporally spaced volumes with different direction diffusion gradients. Each diffusion gradient orientation had 2 separate volumes acquired with opposite-direction $k$-space traversal defined as $k$-left and $k$-right and hence reversed-phase and frequency-encode directions. Acquisitions were cardiacgated to reduce artifacts associated with pulsatile movements of the brain. ${ }^{27}$ Total imaging time was dependent on each participant's heart rate but in the region of 20 minutes for each polarity acquisition.

\section{PET Imaging Acquisitions}

All PET scans were obtained on an ECAT high-resolution research tomograph (HRRT; CTI-Seimens), ${ }^{28,29}$ with a spatial resolution of $2.5-3 \mathrm{~mm}$. A 7-minute transmission scan was performed before tracer injection. ${ }^{18} \mathrm{~F}$ FDG-PET emission data were then acquired in list mode for 60 minutes after intravenous injection of $340 \mathrm{MBq}$ of FDG. Images for the present study were reconstructed as a single frame from data acquired 20-60 minutes postinjection by using iterative ordinary Poisson ordered-subset expectation maximization (10 iterations, 16 subsets), ${ }^{30}$ including corrections for random counts, ${ }^{31}$ scatter, ${ }^{32}$ and attenuation, ${ }^{33}$ with an isotropic voxel size of $1.219 \times 1.219 \times 1.219 \mathrm{~mm}$.

\section{Tissue Segmentation}

With SPM5 (Wellcome Department of Imaging Neuroscience) and Matlab 2007a (MathWorks, Natick, Massachusetts) on a Linux system, all structural T1 images had their origin approximately aligned to the anterior commissure; these T1 images were then segmented by using the unified segmentation algorithm of SPM $5,{ }^{57}$ resulting in probabilistic estimates of gray and white matter tissue classes.

\section{DARTEL Spatial Normalization}

The aligned and segmented GM and WM probability images were entered into the DARTEL toolbox of SPM5; the default settings were used to form the population template. ${ }^{58}$ The flow/warping fields generated by DARTEL were applied to the original individual GM probability images in native space to spatially normalize them to the new DARTEL template. During spatial normalization, images were modulated by their Jacobian determinants to account for the local changes in volume that have occurred as a process of the spatial transformation. The FDG images were initially coregistered to their corresponding $\mathrm{T} 1$ image; these coregistered FDG images were spatially normalized with the flow/ warping fields generated from the DARTEL processing of the GM data; modulation was not performed for the functional FDG data. An 8-mm smoothing kernel was applied to all spatially normalized GM and FDG images.

All DARTEL spatially normalized and modulated GM and spatially normalized and intensity-normalized FDG images (explanation of FDG intensity normalization is given below in the "Region-of-Interest Analysis" section) were analyzed by using the general linear model in SPM5. Because each group was relatively small, regression analyses were performed on the imaging data with the neuropsychological assessments that had revealed the largest group effect (ACE-R Memory, Category Fluency-total score; and ACE-R Attention and Orientation; Table 2) to deter- 
mine the regional relationship among cognition, GM volume, and FDG uptake. For all contrasts, a threshold mask of 0.1 was applied to all images.

Total intracranial volume and age were considered as demographic parameters for covariate inclusion in the statistical analysis of the imaging data. An estimate of total intracranial volume was generated from summing the GM and WM tissue classes, filling the ventricles and dilating the edge of the brain by $3 \mathrm{~mm}$; this procedure was performed with Analyze 7.0 (http://www. analyzedirect.com/support/downloads.asp). Age was included as a confounding covariate because it was not equal across groups. The total intracranial volume measure was not included as a covariate in the FDG voxelwise analysis because total intracranial volume is not expected to be related to regional FDG uptake.

\section{Tract-Based Spatial Statistics Processing and Analysis}

The analysis of the DTI data was performed following the prescribed processing pipeline of voxelwise between-group analysis by using TBSS (Version 1.2). ${ }^{59,60}$ First, diffusion-weighted images were corrected for inhomogeneity artifacts following the procedure outlined in Embleton et al. ${ }^{34}$ The diffusion tensor was then calculated by using the DTIFIT program in FSL (http://fsl.fmrib. ox.ac.uk/fsl/fsl4.0/fdt/fdt_dtifit.html), and the resulting FA 3D volumes were then entered into the TBSS pipeline. All participant FA data were aligned into a common space by using the FMRIB Nonlinear Image Registration Tool based on the most representative scan from the population. After all images had been spatially normalized, a mean FA image was created and a mean FA tract skeleton was derived from it. This skeleton represents the centers of all tracts common to the population. Each participant's aligned FA data were then projected onto this skeleton, and FA values were extracted with the resulting data fed into voxelwise group analysis. We performed the following group contrasts: healthy controls versus $\mathrm{AD}$, healthy controls versus $\mathrm{MCI}$, and $\mathrm{MCI}$ versus $\mathrm{AD}$. For each contrast, a null distribution was constructed from 5000 random permutations. Significance was tested at $P<.05$. The threshold-free cluster enhancement method ${ }^{35}$ was used during analysis, and results were corrected for multiple comparisons.

\section{Region-of-Interest Analysis}

Along with the voxel-based morphometry analyses, a standard region-of-interest investigation was performed in 14 bilateral cortical and 3 subcortical regions (Table 3). The ROIs were derived from the Hammers brain atlas (http://biomedic.doc.ic.ac.uk/ brain-development/index.php?n=Main.AdultMaxProb). ${ }^{61}$ The atlas was warped from Montreal Neurological Institute space into an individual native MR imaging space by using the inverse transformation generated by the segmentation algorithm of SPM5. With Analyze 7.0, each individual GM mask (defined as voxels with > $>50 \% \mathrm{GM}$ probability by SPM5 segmentation) was multiplied by the corresponding Hammers atlas to generate a set of regions containing GM only. The volume of these regions, in percentages relative to total intracranial volume, provided a measure of the regional GM volume. These GM regions were also used to sample the individual regional FDG uptake images, after each individual FDG-PET image was normalized to the median number of counts in the GM of each individual's precentral gyrus. These FDG ratio values are referred to as the FDG uptake.

A region-of-interest investigation was also performed for the FA data. This was performed by using the Johns Hopkins University tract atlas. ${ }^{62}$ This tract atlas is composed of 10 major bilateral white matter tracts. The original Johns Hopkins University tract atlas in Montreal Neurological Institute space was first inverse affine-aligned to the FA volume target image, from the TBSS analysis, by using FSL. Then for each individual participant, an inverse nonlinear warp was estimated by using the invwarp function in FSL (this inverse warp estimates the transformation from the FA volume target image to the original individual FA volume); by applying the inverse warps to the Johns Hopkins University tract atlas, we created individual atlases for each participant in native FA image space. Mean FA values were then measured for each tract atlas region for each individual participant.

Statistical analysis of regional data was performed with the Statistical Package for the Social Sciences, Version 15.0 for Windows (IBM, Armonk, New York). A repeated-measures ANOVA (general linear model with hemisphere and regions as withinparticipant variables and diagnostic group as a between-participant variable, with the Huyn-Feldt correction for nonsphericity if applicable) was used to ensure overall significance, and univariate ANOVA was used for further analysis of individual regions. Age was included as a covariate in all analyses. 